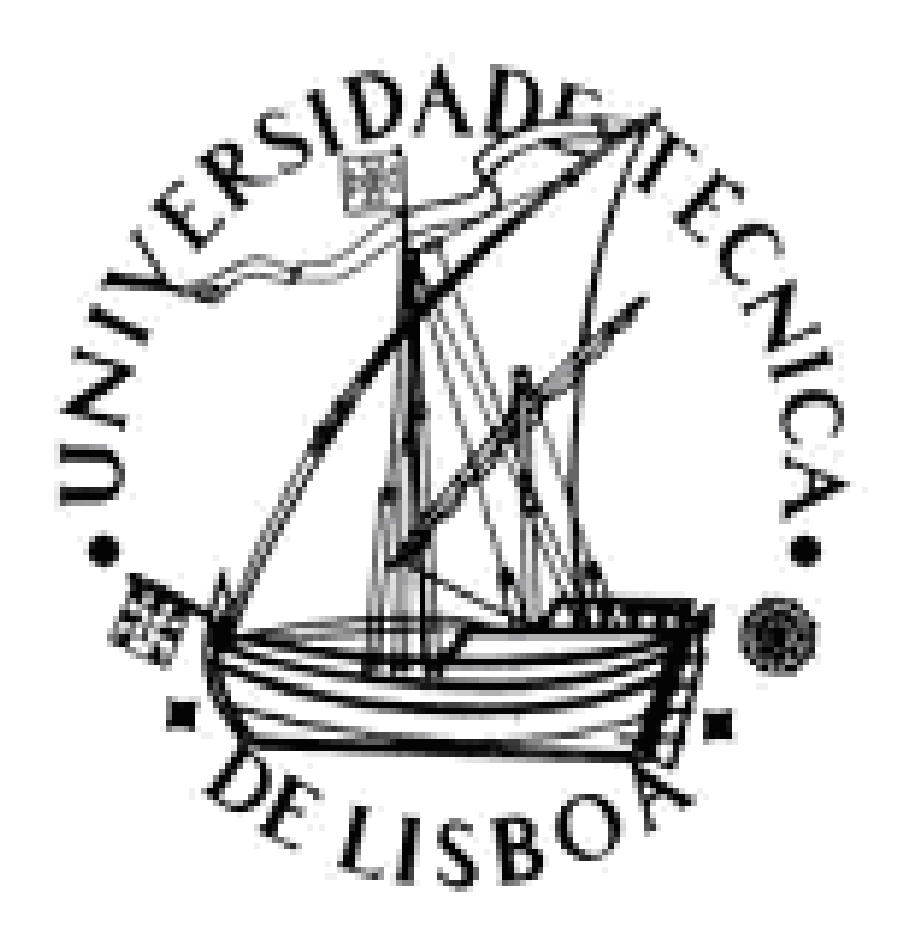

\title{
Sex-Specific Association of Physical Activity with Bone Mass Distribution at the Femoral Neck and Trochanter in Young Adults
}

\author{
Vera Zymbal, Lurdes Rebocho,Graça Cardadeiro, Fátima Baptista
}

\section{INTRODUCTION}

Differences in bone mineral density (BMD) at the proximal femur (PF) may cause differences in incidence of osteoporotic hip fractures between women and men [1]. These differences in BMD may result in part from the skeleton response to mechanical loading by physical activity (PA) which has a positive effect on bone mass [2,3]. Women suffer more fragility fractures in old age [4,5] and have a higher incidence of fractures at the femoral neck region compared to men who have higher incidence of trochanteric femoral fractures [6]. The purpose of this cross-sectional study was to analyze associations between PA and bone mass distribution at the femoral neck and trochanter in young adults.

\section{METHODS}

Subjects. 36 females and 25 male were drawn from university and community by social networks publicity. None of the subjects were taking any medication affecting bone or reported a history of hip fracture. All females were eumenorrheic.

Bone mass distribution at the femoral neck and trochanter. BMD of left leg PF were measured with DXA (QDR Explorer, Hologic, Waltham, MA, USA); BMD ratios among PF subregions were used as surrogates of bone mass distribution and calculated as follows: TR:PF BMD is the ratio between trochanter and PF BMD; neck:PF BMD is the ratio between neck BMD and PF BMD; INF:SUP neck BMD is the ratio between inferomedial and superolateral neck BMD; FN:TR BMD is the ratio between femoral neck and trochanter BMD.

Body size and body composition. Standing height was measured on a stadiometer (Secca 770, Hamburg, Germany) with subjects in underwear and without shoes. Body weight, total fat and total lean mass evaluations were determined from a total body scan by DXA with subjects fasted. Body mass index (BMI) was calculated as body weight in kilogram divided by height in meter squared.

Physical activity was assessed with the Actigraph accelerometer (model GT1M) over seven days and with the Bone-Specific Physical Activity Questionnaire (BPAQ) [7]. Subjects were excluded if they failed to provide a minimum of 4 days of at least 600 minutes per day of accelerometer data. The output from the programme included accumulated time spent at sedentary, light, moderate and vigorous physical activity intensity in minutes per day. The total amount of physical activity was expressed as total counts divided by registered time, that is, counts per minutes, which is an indicator of the intensity physical activity. Cut points of 100, 2020, and 5909 counts/min were current (last 12 months) and past physical activity participation relevant to the musculoskeletal system.

Calcium intake was calculated from a Questionnaire assessing regular intake of dairy products.

\section{Age, Body Composition, Diet and Physical Activity}

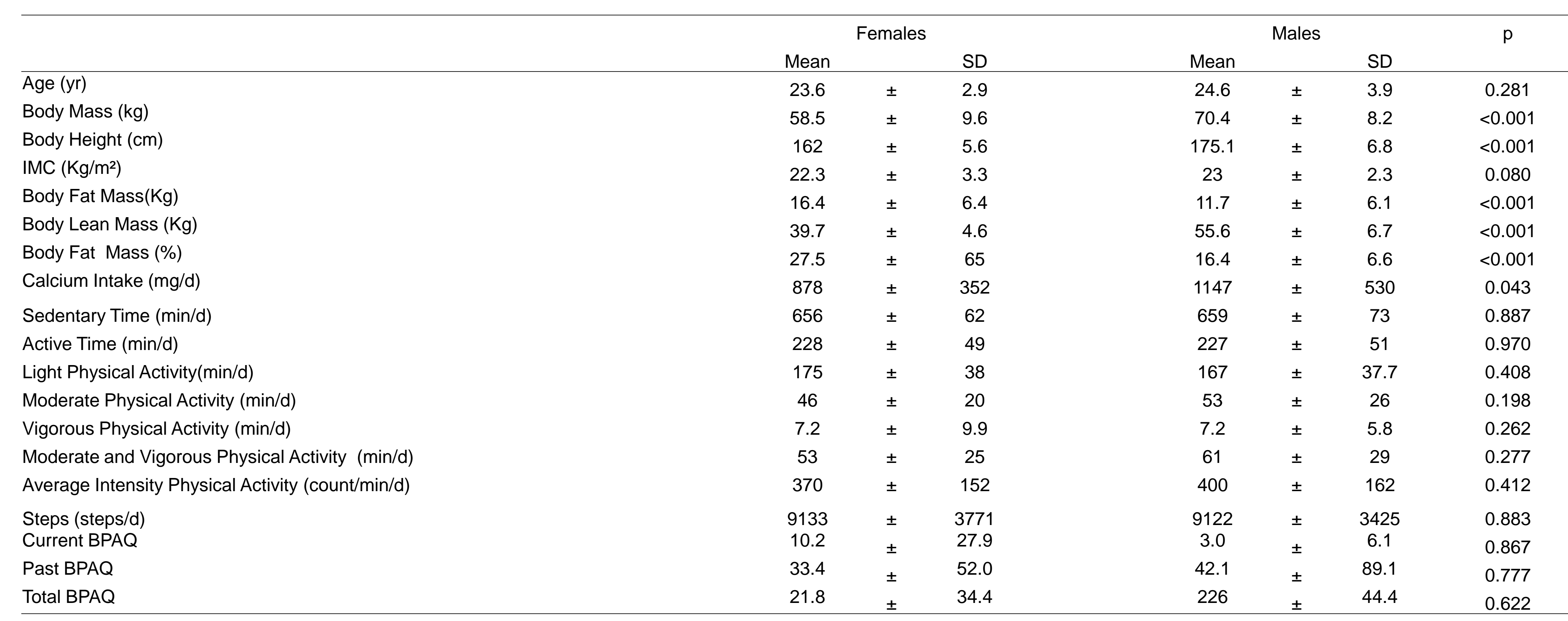
Student's $\mathrm{t}$-test comparing females to males was performed when both variables had normal distribution with the same variance. Student's $\mathrm{t}$-test with Welch
correction was performed when both variables had normal distribution with different variance. In case of no normality Wilcoxon-Mann-Whitney nonparametric test was

\section{BMD and BMD Ratios}

\section{Trochanter BMD (g/comeng
Neck BMD $\left(g / \mathrm{cm}^{2}\right)$}

Superolateral Neck BMD (g/cme)

Inferomedial Neck BMD $\left(g / \mathrm{cm}^{2}\right)$

TR:PF BMD

Neck:PF BMD

INF:SUP Neck BM

FN:TR BMD

Student's t-test comparing females to males was performed when both variables had normal distribution with the same variance. Student's $\mathrm{t}$-test with Welch
correction was performed when both variables had normal distribution with different variance. In case of no normality Wilcoxon-Mann-Whitney nonparametric test was

Funded by Portuguese Science and Technology Foundation PTDC/DES/115607/2009 and PhD Scholarship SFRH / BD /

\begin{tabular}{|c|c|c|c|c|c|c|c|c|}
\hline & \multicolumn{2}{|c|}{ Trochanter BMD } & \multicolumn{2}{|c|}{ Neck BMD } & \multicolumn{2}{|c|}{ Superolateral Neck BMD } & \multicolumn{2}{|c|}{ Inferomedial Neck BMD } \\
\hline & females & males & females & males & & & & males \\
\hline Sedentary Time & 0.233 & -0.149 & 0.035 & -0.005 & 0.059 & 0.070 & 0.011 & -0.093 \\
\hline Ictive Time & -0.293 & 0.250 & -0.121 & 0.085 & -0.125 & -0.042 & -0.098 & 0.224 \\
\hline ight Physical Activity & $-0.371^{*}, 0$ & 0.079 & -0.241 & -0.050 & -0.214 & -0.201 & -0.220 & 0.124 \\
\hline Moderate Physical Activity & -0.042 & 0.252 & 0.088 & 0.129 & 0.045 & 0.103 & 0.105 & 0.154 \\
\hline Iigorous Physical Activity & -0.072 & 0.524 * & 0.155 & $0.493^{*}$ & 0.117 & $0.489^{\circ}$ & 0.159 & 0.441 \\
\hline oderate and Vigorous Physical Activity & -0.004 & 0.330 & 0.131 & 0.214 & 0.083 & 0.191 & 0.146 & 0.226 \\
\hline iverage Intensity of Physical Activity & 0.006 & 0.339 & 0.135 & 0.221 & 0.060 & 0.184 & 0.170 & 0.241 \\
\hline iteps & -0.202 & 0.393 & -0.006 & 0.259 & -0.039 & 0.205 & 0.012 & 0.301 \\
\hline Surrent $B P A Q$ & $0.430^{\circ}$ & 0.267 & $0.453^{*}$ & 0.164 & $0.439^{*}$ & 0.205 & $0.448^{\circ}$ & 0.108 \\
\hline Past BPAQ & 0.234 & -0.097 & 0.112 & -0.149 & 0.159 & -0.127 & 0.073 & -0.149 \\
\hline tatal BPAQ & 0.351 & -0.081 & 0.269 & -0.140 & 0.299 & -0.115 & 0.237 & -0.144 \\
\hline \multicolumn{9}{|c|}{$p<0.05 ; "$ p $<0.01$ - Adiusted for Body Mass and Body Height } \\
\hline & \multirow{2}{*}{\multicolumn{2}{|c|}{$\begin{array}{c}\text { TR:PF BMD } \\
\text { females }\end{array}$}} & \multirow{2}{*}{\multicolumn{2}{|c|}{ Neck:PF BMD }} & \multirow{2}{*}{\multicolumn{2}{|c|}{$\begin{array}{l}\text { INF:SUP Neck BMD } \\
\text { tomac }\end{array}$}} & \multicolumn{2}{|c|}{ FN:TR BMD } \\
\hline & & & & & & & females & males \\
\hline intary Time & 0.305 & -0.139 & -0.159 & $0.440^{*}$ & -0.042 & -0.246 & -0.330 & 0.401 \\
\hline Ictive Time & -0.279 & 0.171 & 0.111 & $-0.550^{* *}>0$ & 0.058 & 0.366 & 0.281 & $-0.513^{*}+4$ \\
\hline ight Physical Activity & -0.311 & 0.000 & -0.009 & $-0.573^{* *}$ & 0.070 & $0.518^{\circ}$ & 0.207 & $-0.4311^{*} \times$ \\
\hline Moderate Physical Activity & -0.047 & 0.290 & 0.205 & -0.213 & 0.024 & 0.026 & 0.197 & -0.329 \\
\hline Iigorous Physical Activity & -0.060 & 0.204 & 0.164 & -0.073 & -0.036 & -0.335 & 0.175 & -0.173 \\
\hline Moderate and Vigorous Physical Activity & -0.061 & 0.299 & 0.228 & -0.203 & 0.005 & -0.046 & 0.226 & -0.327 \\
\hline iverage Intensity of Physical Activity & -0.123 & 0.260 & 0.181 & -0.250 & 0.065 & 0.006 & 0.226 & -0.336 \\
\hline iteps & -0.200 & 0.292 & 0.208 & -0.263 & 0.047 & 0.010 & 0.304 & -0.367 \\
\hline Eurrent $B P A Q$ & 0.273 & $0.579^{*}$ & 0.323 & 0.040 & -0.133 & -0.225 & 0.070 & -0.319 \\
\hline Past BPAQ & $0.353^{*} \times$ & 0.001 & 0.072 & -0.248 & -0.156 & 0.054 & -0.180 & -0.195 \\
\hline & & 0.041 & 0.186 & -0.246 & -0.172 & 0.039 & -0.107 & -0.218 \\
\hline
\end{tabular}

\section{Regression Analysis}
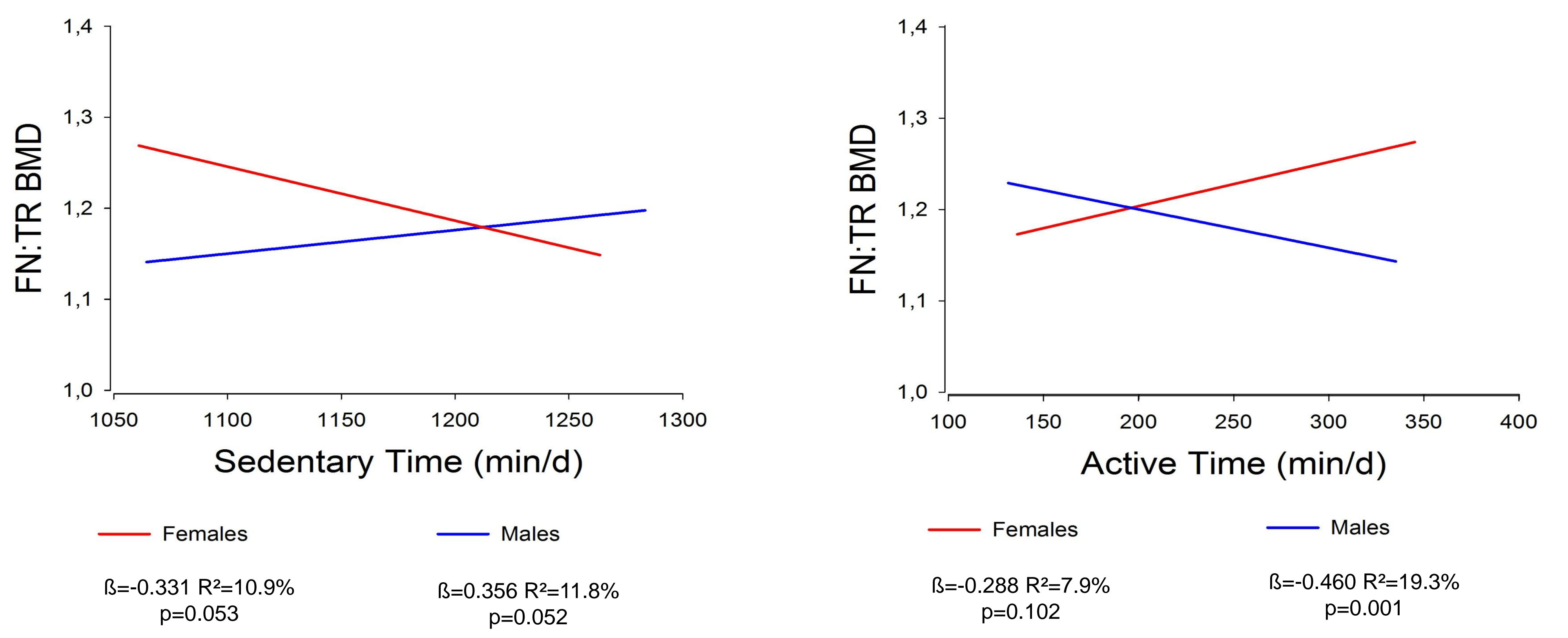

CONCLUSIONS

Positive associations between PA and BMD of the trochanter, inferomedial, superolateral, and integral femoral neck in both genders: the vigorous PA in males and the BPAQ in females were the most significant PA variables.

Compared with other regions of proximal femur, increased physical activity favors more the trochanter in both genders and sedentary time favors more the femoral neck in males.

In females it was not found significant associations of FN:TR BMD and PA variables, despite a trend in the association of FN:TR BMD with sedentary time $(r=-0.330, p=0.053)$ and with steps/day $(r=0.304, p=0.075)$.

* PA seems to be related with bone mass distribution in males with a more active lifestyle (independent of PA intensity) to favor the BMD of the trochanteric region and a light PA to favor the inferomedial region of the femoral neck. The association in females appear to have a contrary relationship with a more active lifestyle to promote the femoral neck compared to the trochanteric region.

\section{REFERENCES}

[1] Peacock, M., Buckwalter, K. A., Persohn, S., Hang 2] Schwab, P., \& Scalapino, K. (2011). Exercise for bone health: rationale and prescription. Curr Opin Rheumatol, 23, 137-141.

[3] Seeman, E. (2008). Bone quality: the material and structural basis of bone strength. J Bone Miner Metab, 26, 1-8.

[4] Shao, C. J., Hsieh, Y. H., Tsai, C. H., \& Lai, K. A. (2009). A nationwide seven-year trend of hip fractures in the elderly population of Taiwan. Bone, 44, 125-129.

[5] El Maghraoul, A., Koumba, B. A., Jroundi, I., Achemlal, L., Bezza, A., \& Tazi, M. A. (2005). Epidemiology of hip fractures in 2002 in Rabat,

[6] Lin, W. P., Wen, C. J., Jiang, C. C., Hou, S. M., Chen, C. Y., \& Lin, J. (2011). Risk factors for hip fracture sites and mortality in older adults. J 7] Weeks, B. K., \& Beck, B. R. (2008). The BPAQ: a bone-specific physical activity assessment instrument. Osteoporos Int, 19, 1567-1577. [8] Baptista F, Santos DA, Silva AM, Mota J, Santos R, Vale S, Ferreira JP, Raimundo AM, Moreira H, Sardinha LB (2012) Prevalence of the Portuguese population attaining sufficient physical activity. Med Sci Sports Exerc, 44, 466-73. 